\title{
Avaliação de três métodos radiográficos (periapical convencional, periapical digital e panorâmico) no diagnóstico de lesões apicais produzidas artificialmente
}

\section{Evaluation of three radiographic methods (conventional periapical, digital periapical and panoramic) in the diagnosis of artificially produced periapical lesions}

Solange Maria de ALMEIDA*

Frab Norberto BÓSCOLO**

Francisco HAITER NETO***

Júlio César Bento dos SANTOS****

\begin{abstract}
ALMEIDA, S. M. de; BÓSCOLO, F. N.; HAITER NETO, F.; SANTOS, J. C. B. dos. Avaliação de três métodos radiográfi$\cos$ (periapical convencional, periapical digital e panorâmico) no diagnóstico de lesões apicais produzidas artificialmente. Pesqui Odontol Bras, v. 15, n. 1, p. 56-63, jan./mar. 2001.
\end{abstract}

\begin{abstract}
Esta pesquisa teve por objetivo avaliar três métodos radiográficos - radiografia periapical convencional, periapical digital e panorâmica - no diagnóstico de lesões apicais produzidas artificialmente. Para tanto, utilizaram-se mandíbulas maceradas, onde foram produzidas lesões com brocas esféricas de diferentes diâmetros, permitindo que as lesões apresentassem tamanhos diferentes, conforme a broca utilizada, o que correspondeu a 5 fases distintas: fase inicial ou $Z$, caracterizada pela ausência de lesão; fase R, cuja lesão foi produzida com a broca 6; fase J, lesão produzida com a broca 8; fase D, cuja lesão foi produzida com a broca 10; fase $\mathrm{H}$, cuja destruição óssea atingia a cortical vestibular. As lesões foram produzidas em todos os quadrantes dentais. As radiografias foram realizadas após cada fase e analisadas por 4 radiologistas. Houve diferença estatisticamente significante na fase $\mathrm{R}$ na região de incisivos e na fase $\mathrm{H}$ na região de pré-molares a favor do sistema digital; na região de molares houve diferença estatisticamente significante na fase $\mathrm{D}$ para a radiografia panorâmica, sendo que essa técnica foi a menos eficaz na fase $\mathrm{H}$.
\end{abstract}

UNITERMOS: Abscesso periapical; Intensificação de imagem radiográfica; Radiografia panorâmica; Radiografia dentária.

\section{INTRODUÇÃO}

A condição radiográfica do periápice radicular é extremamente importante para o estabelecimento do plano de tratamento endodôntico e/ou cirúrgico. Contudo, as dificuldades no diagnóstico foram descritas em um trabalho pioneiro de SELTZER; $\operatorname{BENDER}^{13,14}$ (1961), demonstrando que lesões confinadas ao osso esponjoso eram imperceptiveis radiograficamente, a menos que se estendessem às corticais vestibular, lingual ou a ambas. Segundo os autores, grandes lesões intra-ósseas, de origem inflamatória ou tumoral, podem estar presentes, sem apresentar alteração na característica radiográfica. PAULS; TROTT ${ }^{8}$ (1966) produziram lesões apicais em mandíbulas humanas maceradas, utilizando brocas esféricas de vários diâmetros, con- firmando os resultados obtidos por SELTZER; $\operatorname{BENDER}^{13,14}$ (1961).

Vários estudos têm sido realizados com o objetivo de avaliar o valor do exame por imagem no diagnóstico de lesões apicais ${ }^{2,4,5,15,16,17}$. Alguns trabalhos mostraram superioridade dos sistemas digitais ${ }^{4,15,16}$. Um outro estudo mostrou que depende do tamanho da lesão, visto que, quando não existe lesão, a radiografia periapical é melhor, já quando a lesão atinge as corticais, ela é melhor visualizada na radiografia digital ${ }^{17}$. Quando comparadas imagens digitais originais e manipuladas, não há diferença ${ }^{5}$.

Segundo PHILLIPS; SHAWKAT ${ }^{10}$ (1973), as radiografias panorâmicas são mais eficientes que as radiografias periapicais na avaliação de lesões api-

* Professora Doutora; ** Professor Titular; *** Professor Livre-Docente - Faculdade de Odontologia de Piracicaba da UNICAMP. **** Professor Assistente de Radiologia da Faculdade de Odontologia de Montes Claros - MG. 
ALMEIDA, S. M. de; BÓSCOLO, F. N.; HAITER NETO, F.; SANTOS, J. C. B. dos. Avaliação de três métodos radiográficos (periapical convencional, periapical digital e panorâmico) no diagnóstico de lesões apicais produzidas artificialmente. Pesqui Odontol Bras, v. 15 , n. 1, p. 56-63, jan./mar. 2001.

cais produzidas artificialmente. ROHLIN et al. ${ }^{11}$ (1989) demonstraram não haver diferença estatisticamente significante entre esses dois procedimentos, embora em algumas áreas específicas uma das técnicas tenha demonstrado melhores resultados.

No diagnóstico de lesões apicais, GRÖNDAL et $a l^{3}$ (1996) observaram não haver diferença entre o sistema Digora e o filme Ektaspeed. Já BARBAT; MESSER $^{1}$ (1998) afirmaram também não haver diferença estatisticamente significante entre o filme Ultraspeed e o sistema digital Digora quando utilizaram recursos como imagem reversa, cor e escala de cinza.

KULLENDORFF et al. ${ }^{6}$ (1998), comparando subtração de imagem e radiografia periapical, observaram diferença estatisticamente significante em relação à subtração na detecção de lesões periapicais precoces, entretanto quando a lesão apresentava maior dimensão, os dois métodos não apresentavam diferença estatística.

Em 1999, MARMARY et al. ${ }^{7}$ realizaram um estudo que teve por objetivo determinar se é necessário ocorrer a destruição da cortical óssea para que a lesão seja observada radiograficamente. Após as lesões serem detectadas, foi realizado exame por tomografia computadorizada, onde das 42 lesões que foram detectadas por radiografias periapicais, 31 lesões mostraram, através dos cortes seccionais, não atingirem as lâminas ósseas corticais.

Também em 1999, SCARFE et al. ${ }^{12}$ compararam as dimensões de 25 lesões entre radiografia convencional (filme E-speed Plus) e imagem digital (sistema CCD - imagem não ampliada, equalizada e equalizada/colorida) com o tamanho real, medido quando da cirurgia. Foi observado que o tamanho real das lesões foi maior que o dos dois métodos de exame. Quando utilizado o sistema de cor, este foi menos preciso que a imagem equalizada e imagem não ampliada. As medidas realizadas pela régua do sistema digital foram mais precisas que por régua comum nas radiografias convencionais.

PAURAZAS et al. ${ }^{9}$ (2000) compararam a precisão de diagnóstico na detecção de lesões apicais produzidas artificialmente no osso cortical e medular, entre três métodos: radiografia convencional (filme E-speed Plus), imagem digital (sistema CCD) e um sensor de pixel ativo semicondutor complementar metal-óxido (CMOS-APS). Não foi encontrada diferença estatística entre os três métodos. Em relação aos dois tipos de tecido ósseo, a precisão da detecção de lesão no osso cortical foi significantemente maior do que no osso medular.
Diante das controvérsias existentes, decidiu-se comparar três métodos radiográficos: radiografia periapical convencional, radiografia periapical digital e radiografia panorâmica, no sentido de observar qual método detecta mais precocemente lesões periapicais produzidas artificialmente e se o tamanho da lesão interfere no diagnóstico radiográfico.

\section{MATERIAIS E MÉTODOS}

Após exame radiográfico, foram selecionadas cinco mandíbulas humanas maceradas, com um número variável de dentes, mas apresentando todos os grupos dentais: incisivos, caninos, pré-molares e molares, sendo descartadas aquelas que apresentavam dentes com lesão apical ou condições que a simulassem.

As mandíbulas foram imersas em água para facilitar a remoção dos dentes, que, após terem sido removidos, foram recolocados nos respectivos alvéolos e radiografados. As lesões foram produzidas utilizando-se brocas esféricas de baixa rotação de números 6,8 , e 10 . Portanto, o diâmetro de cada lesão correspondia a cada broca utilizada, sendo que a última fase correspondeu àquela em que o desgaste ósseo provocado pela brocas atingiu a cortical vestibular. A cada aumento da lesão o dente era recolocado no alvéolo e radiografado, tendose o cuidado de padronizar a posição da mandíbula em cada tomada radiográfica. Para identificar-se cada etapa da pesquisa, a fase inicial foi considerada como sendo aquela em que não havia lesão apical, denominada aleatoriamente de fase $Z$, a fase $\mathrm{R}$ correspondia a lesão produzida com a broca 6 , a fase $\mathrm{J}$ correspondia a lesão com a broca 8 , a fase D seria a lesão com a broca 10 e a fase H correspondia à lesão cuja destruição óssea tocava a cortical vestibular (Figura 1).

Para simular tecido mole, foi utilizada uma camada de $5 \mathrm{~mm}$ de silicone na face externa de cada mandíbula.

O material utilizado foi o seguinte:

- Filmes: foram utilizados para obtenção das radiografias periapicais convencionais o filme EP-21p, Ektaspeed Plus (Eastman Kodak Company). Para as radiografias panorâmicas, utilizaram-se os filmes T-Mat (Kodak do Brasil) com "écran" intensificador Kodak Ektavision (X-omatic).

- Sensor digital: a imagem digital foi obtida com placa de fósforo.

- Aparelhos de raios X: para a obtenção das ima- 
ALMEIDA, S. M. de; BÓSCOLO, F. N.; HAITER NETO, F.; SANTOS, J. C. B. dos. Avaliação de três métodos radiográficos (periapical convencional, periapical digital e panorâmico) no diagnóstico de lesões apicais produzidas artificialmente. Pesqui Odontol Bras, v. 15 , n. 1, p. 56-63, jan./mar. 2001.

gens radiográficas periapicais, convencionais e digitais, utilizou-se o aparelho GE 1000 (General Eletric Company, Milwaukee, Wisconsin, EUA), com os seguintes fatores: $65 \mathrm{kVp}, 10 \mathrm{~mA}$ e um tempo de exposição de 0,4 segundos, sendo que para a radiografia digital, esse tempo de exposição foi reduzido à metade. Para as radio-
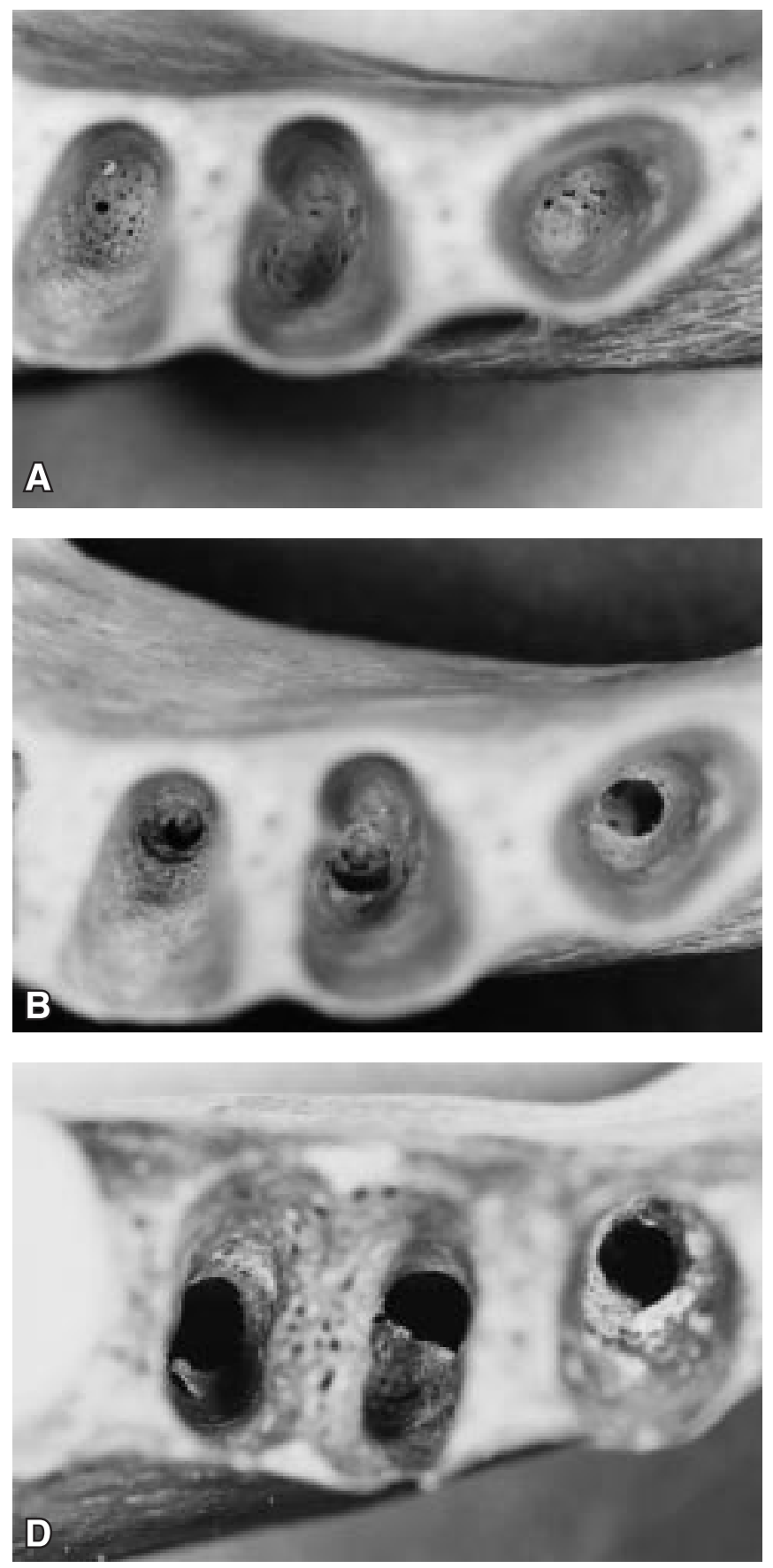

grafias panorâmicas, utilizou-se o Orthopantomographic OP 100 (Instrumentarium Corp. Imaging Division, Finlândia), operando com $57 \mathrm{kVp}$ e $2 \mathrm{~mA}$.

- Processamento: o processamento dos filmes foi realizado em processadoras automáticas (Macrotec MX2 e GENDEX GXP). A imagem digital foi obtida no "scanner" Digora (Soredex-Finndent, Orion Corporation, Helsinki, Finlândia).

Uma vez obtidas as imagens, estas foram avaliadas por quatro profissionais com mestrado em Radiologia. Antes de iniciar a interpretação, cada examinador recebeu informações de que poderia haver ou não lesão apical produzida por instrumentos rotatórios de diferentes diâmetros.

Os examinadores avaliaram cada técnica radiográfica em dias alternados, para que não houvesse comparação entre as três técnicas empregadas. A avaliação foi realizada mediante a atribuição dos seguintes escores: 1 - lesão ausente; 2 - lesão provavelmente ausente; 3 - lesão provavelmente presente; 4 - lesão presente.
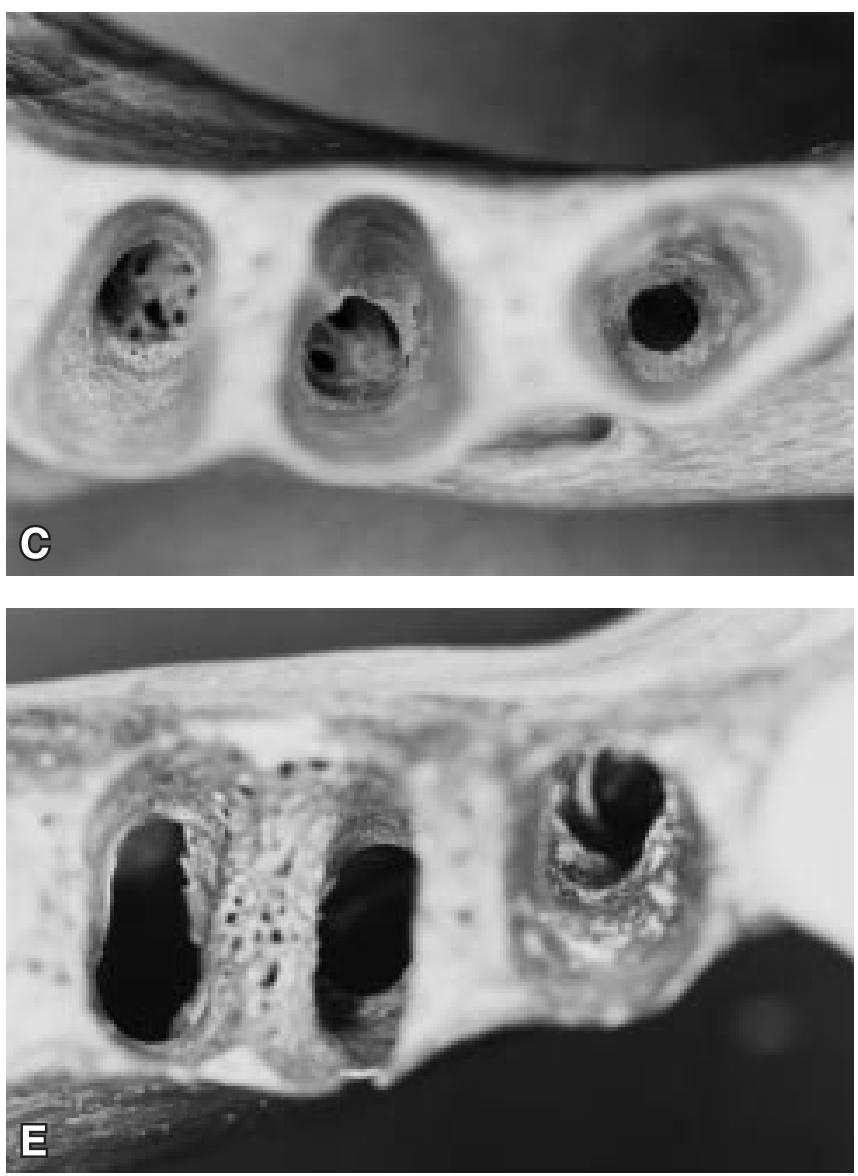

FIGURA 1 - Lesões ósseas produzidas artificialmente: inicial (A); broca 6 (B); broca 8 (C); broca 10 (D); cortical (E). 
ALMEIDA, S. M. de; BÓSCOLO, F. N.; HAITER NETO, F.; SANTOS, J. C. B. dos. Avaliação de três métodos radiográficos (periapical convencional, periapical digital e panorâmico) no diagnóstico de lesões apicais produzidas artificialmente. Pesqui Odontol Bras, v. 15, n. 1, p. 56-63, jan./mar. 2001.

TABELA 1 - Médias das notas conferidas às imagens radiográficas, segundo a técnica, para cada fase na região de incisivos.

\begin{tabular}{l|c|c|c|c|c}
\hline \hline Técnica & $\begin{array}{c}\text { Inicial } \\
(\mathrm{Z})\end{array}$ & $\begin{array}{c}\text { Broca 6 } \\
(\mathrm{R})\end{array}$ & $\begin{array}{c}\text { Broca 8 } \\
(\mathrm{J})\end{array}$ & $\begin{array}{c}\text { Broca 10 } \\
(\mathrm{D})\end{array}$ & $\begin{array}{c}\text { Cortical } \\
(\mathrm{H})\end{array}$ \\
\hline Periapical & $1,75 \mathrm{~A}$ & $1,60 \mathrm{~B}$ & $2,00 \mathrm{~A}$ & $2,35 \mathrm{~A}$ & $3,35 \mathrm{~A}$ \\
\hline Digital & $1,60 \mathrm{~A}$ & $2,30 \mathrm{~A}$ & $1,80 \mathrm{~A}$ & $2,45 \mathrm{~A}$ & $3,55 \mathrm{~A}$ \\
\hline Panorâmica & $1,20 \mathrm{~A}$ & $1,65 \mathrm{AB}$ & $1,75 \mathrm{~A}$ & $2,20 \mathrm{~A}$ & $3,20 \mathrm{~A}$ \\
\hline \hline
\end{tabular}

$\mathrm{Dp}(Z)=0,08319 ; \mathrm{dp}(\mathrm{R})=0,9212 ; \mathrm{dp}(\mathrm{J})=0,9554 ; \mathrm{dp}$ (D) $=0,9844 ; \mathrm{dp}(\mathrm{H})=0,82$. Letras diferentes indicam diferença estatística.

TABELA 2 - Médias das notas conferidas às imagens radiográficas, segundo a técnica, para cada fase na região de caninos.

\begin{tabular}{l|c|c|c|c|c}
\hline \hline Técnica & $\begin{array}{c}\text { Inicial } \\
(\mathrm{Z})\end{array}$ & $\begin{array}{c}\text { Broca 6 } \\
(\mathrm{R})\end{array}$ & $\begin{array}{c}\text { Broca } 8 \\
(\mathrm{~J})\end{array}$ & $\begin{array}{c}\text { Broca 10 } \\
(\mathrm{D})\end{array}$ & $\begin{array}{c}\text { Cortical } \\
(\mathrm{H})\end{array}$ \\
\hline Periapical & $1,812 \mathrm{~A}$ & $1,500 \mathrm{~A}$ & $2,312 \mathrm{~A}$ & $2,625 \mathrm{~A}$ & $3,500 \mathrm{~A}$ \\
\hline Digital & $1,750 \mathrm{~A}$ & $1,562 \mathrm{~A}$ & $2,250 \mathrm{~A}$ & $2,625 \mathrm{~A}$ & $3,187 \mathrm{~A}$ \\
\hline Panorâmica & $1,750 \mathrm{~A}$ & $1,875 \mathrm{~A}$ & $2,315 \mathrm{~A}$ & $2,875 \mathrm{~A}$ & $3,125 \mathrm{~A}$ \\
\hline \hline
\end{tabular}

$\mathrm{Dp}(Z)=0,9925 ; \mathrm{dp}(\mathrm{R})=0,7552 ; \mathrm{dp}(\mathrm{J})=0,8880 ; \mathrm{dp}$ (D) $=0,8521 ; \mathrm{dp}(\mathrm{H})=0,8539$. Letras diferentes indicam diferença estatística.

\section{RESULTADOS}

As notas conferidas às imagens radiográficas pelos quatro avaliadores foram submetidas à análise de variância, para comparar técnicas. Foi feita uma análise para cada fase de cada região usando avaliadores como réplicas. Os resultados estão apresentados em seguida.

As médias das notas, segundo cada técnica, estão apresentadas nas Tabelas 1, 2, 3 e 4. Estas médias foram comparadas pelo teste de Tukey.

\section{Região de incisivos}

Considerando-se que as médias das notas indicam a facilidade de observação oferecida pela imagem radiográfica e considerando-se os resultados do teste de Tukey apresentados na Tabela 1, conclui-se que, para a região de incisivos (Figura 2), os resultados obtidos pela radiografia digital são, em média, melhores quando se usa a broca 6 (fase R).

\section{Região de caninos}

Considerando-se que as médias das notas indicam a facilidade de observação oferecida pela imagem radiográfica e considerando-se os resultados
TABELA 3 - Médias das notas conferidas às imagens radiográficas, segundo a técnica, para cada fase na região de pré-molares.

\begin{tabular}{l|c|c|c|c|c}
\hline \hline Técnica & $\begin{array}{c}\text { Inicial } \\
(\mathrm{Z})\end{array}$ & $\begin{array}{c}\text { Broca 6 } \\
(\mathrm{R})\end{array}$ & $\begin{array}{c}\text { Broca 8 } \\
(\mathrm{J})\end{array}$ & $\begin{array}{c}\text { Broca 10 } \\
(\mathrm{D})\end{array}$ & $\begin{array}{c}\text { Cortical } \\
(\mathrm{H})\end{array}$ \\
\hline Periapical & $2,214 \mathrm{~A}$ & $2,214 \mathrm{~A}$ & $2,464 \mathrm{~A}$ & $2,821 \mathrm{~A}$ & $3,679 \mathrm{AB}$ \\
\hline Digital & $2,179 \mathrm{~A}$ & $2,250 \mathrm{~A}$ & $2,536 \mathrm{~A}$ & $2,857 \mathrm{~A}$ & $3,821 \mathrm{~A}$ \\
\hline Panorâmica & $1,786 \mathrm{~A}$ & $2,143 \mathrm{~A}$ & $2,179 \mathrm{~A}$ & $2,357 \mathrm{~A}$ & $3,500 \mathrm{~B}$ \\
\hline \hline
\end{tabular}

$\operatorname{Dp}(Z)=0,9925 ; \mathrm{dp}(\mathrm{R})=0,8621 ; \mathrm{dp}(\mathrm{J})=0,8634 ; \mathrm{dp}$ (D) $=0,9071 ; \mathrm{dp}(\mathrm{H})=0,6770$. Letras diferentes indicam diferença estatística.

TABELA 4 - Médias das notas conferidas às imagens radiográficas, segundo a técnica, para cada fase na região de molares.

\begin{tabular}{l|c|c|c|c|c}
\hline \hline Técnica & $\begin{array}{c}\text { Inicial } \\
(\mathrm{Z})\end{array}$ & $\begin{array}{c}\text { Broca 6 } \\
(\mathrm{R})\end{array}$ & $\begin{array}{c}\text { Broca } 8 \\
(\mathrm{~J})\end{array}$ & $\begin{array}{c}\text { Broca 10 } \\
(\mathrm{D})\end{array}$ & $\begin{array}{c}\text { Cortical } \\
(\mathrm{H})\end{array}$ \\
\hline Periapical & $2,031 \mathrm{~A}$ & $1,125 \mathrm{~A}$ & $1,125 \mathrm{~A}$ & $1,875 \mathrm{~B}$ & $3,812 \mathrm{~A}$ \\
\hline Digital & $1,469 \mathrm{~B}$ & $1,281 \mathrm{~A}$ & $1,250 \mathrm{~A}$ & $2,000 \mathrm{~B}$ & $3,687 \mathrm{~A}$ \\
\hline Panorâmica & $1,312 \mathrm{~B}$ & $1,344 \mathrm{~A}$ & $1,437 \mathrm{~A}$ & $2,687 \mathrm{~A}$ & $3,375 \mathrm{~B}$ \\
\hline \hline
\end{tabular}

$\mathrm{Dp}(\mathrm{Z})=0,8750 ; \mathrm{dp}(\mathrm{R})=0,6070 ; \mathrm{dp}(\mathrm{J})=0,6326 ; \mathrm{dp}$ $(D)=1,1228 ; \mathrm{dp}(\mathrm{H})=0,5162$. Letras diferentes indicam diferença estatística.

do teste de Tukey apresentados na Tabela 2, conclui-se que para a região de caninos não se verificou diferença estatística entre as técnicas.

\section{Região de pré-molares}

Considerando-se que as médias das notas indicam a facilidade de observação oferecida pela imagem radiográfica e considerando-se os resultados do teste de Tukey apresentados na Tabela 3, conclui-se que, para a região dos dentes pré-molares, o resultado obtido pela radiografia digital é ligeiramente melhor do que o obtido pela panorâmica na fase cortical.

\section{Região de molares}

Considerando-se que as médias das notas indicam a facilidade de observação oferecida pela imagem radiográfica e considerando-se os resultados do teste de Tukey apresentados na Tabela 4, conclui-se que, na região de molares (Figura 3), para a fase $Z$ (inicial), os resultados obtidos pela radiografia periapical são, em média, melhores que os obtidos pelas outras; para a fase D (broca 10), os resultados obtidos pela radiografia panorâmica são, em 
ALMEIDA, S. M. de; BÓSCOLO, F. N.; HAITER NETO, F.; SANTOS, J. C. B. dos. Avaliação de três métodos radiográficos (periapical convencional, periapical digital e panorâmico) no diagnóstico de lesões apicais produzidas artificialmente. Pesqui Odontol Bras, v. 15 , n. 1, p. 56-63, jan./mar. 2001.

média, melhores que os obtidos pelas outras e para a fase $\mathrm{H}$ (cortical), os resultados obtidos pelas radiografias digital e periapical são, em média, melhores que os obtidos pela panorâmica.

\section{DISCUSSÃO}

Quando avaliada a região dos dentes anteriores, observa-se que não houve diferença estatisticamente significante entre os três métodos radiográficos nas fases $Z, J, D$ e $H$. No entanto, observa-se que a radiografia digital foi superior aos outros métodos para a observação da lesão na fase R. Embora alguns estudos indiquem a superioridade dos sistemas digitais ${ }^{3,10,11}$ em relação ao método convencional, observa-se que neste estudo essa superioridade ocorreu somente na fase R. Equivalência na detecção de lesões apicais, entre o filme e o sistema digital, também foi encontrada no trabalho de YOKOTA et al. ${ }^{17}$ (1994), GRÖNDAHL et al. ${ }^{3}$ (1996), BARBAT; MESSER ${ }^{1}$ (1998) e PAURAZAS et al. ${ }^{9}$ (2000). Isso pode ser justificado pelo fato de que a radiografia digital apresenta melhor baixo contraste, o que permite avaliar nuanças de cinza
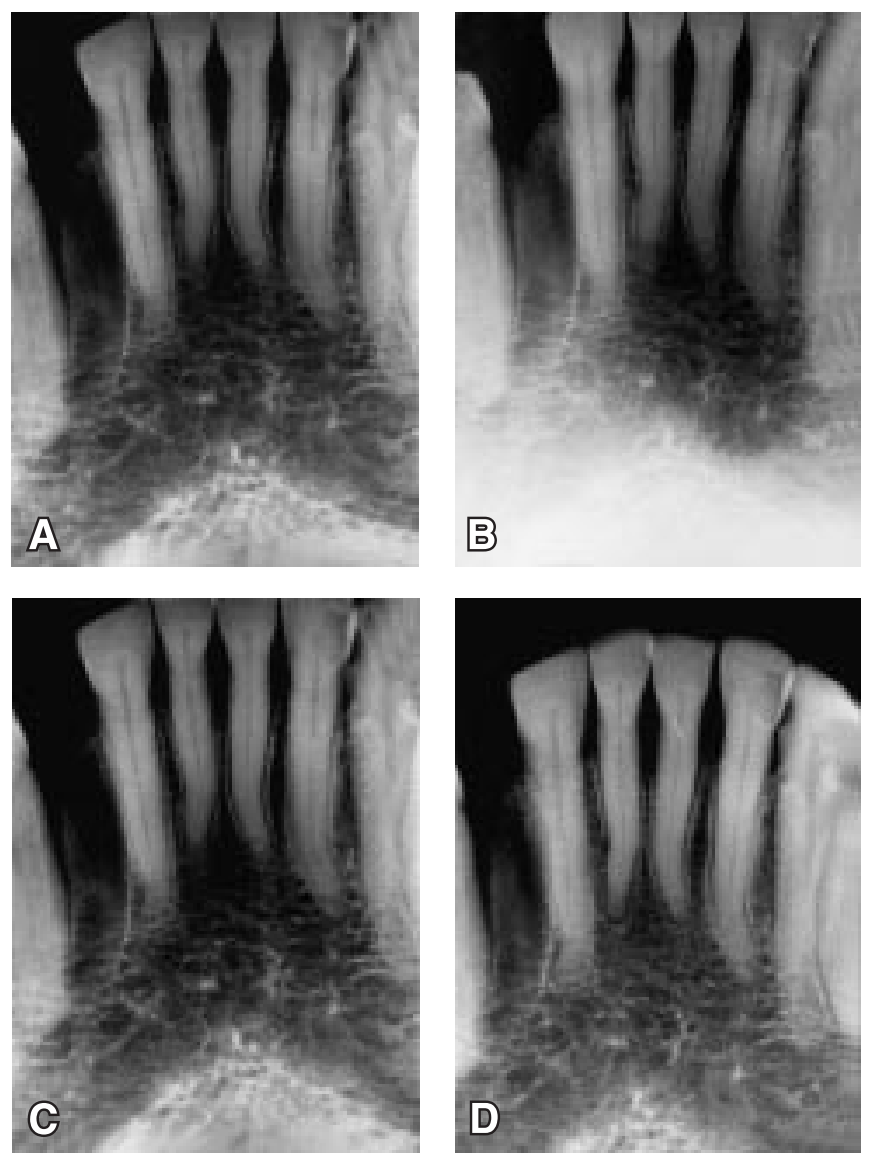

mais facilmente, devido a uma escala dinâmica maior quando comparada ao filme periapical convencional. Concorda-se, portanto, com WENZEL ${ }^{16}$ (1993) quando a autora afirma que a técnica digital é particularmente útil para detectar pequenas mudanças no osso alveolar.

Analisando-se a região de caninos, observa-se não haver diferença estatisticamente significante entre técnicas e fases, demonstrando que a situação foi bem avaliada em todas as fases e em todas as técnicas. Percebeu-se que, à medida que o diâmetro de lesão aumentou, as médias das notas também aumentaram, o que indica que quando a lesão atingiu a cortical óssea, assumindo portanto, um maior diâmetro, a facilidade em observar sua imagem na radiografia também aumentou. Isso pode ser devido ao fato de que a região de canino é uma região onde a espessura óssea é mais reduzida, contribuindo, portanto, para melhor visualização das condições reais desta região.

Para a região dos pré-molares, observa-se que a radiografia panorâmica foi a técnica que mostrou o pior resultado para visualizar a lesão produzida, em todas as fases do experimento. ROHLIN et al. ${ }^{11}$ (1989) chegaram à mesma conclusão quando trabalharam com radiografias periapicais e panorâmicas para estudo de lesões apicais. Os autores atribuíram a dificuldade do diagnóstico das lesões apicais nas radiografias panorâmicas, à sobreposição das estruturas e à formação de sombras, principalmente da coluna vertebral e do crânio.

Neste trabalho, observa-se que as sobreposições de imagem de estruturas não interferiu, visto que a mandíbula foi radiografada de forma isolada; entretanto, não se pode esquecer que a radiografia panorâmica é um método tomográfico, sendo a imagem produzida em cortes. Ainda nesta região,

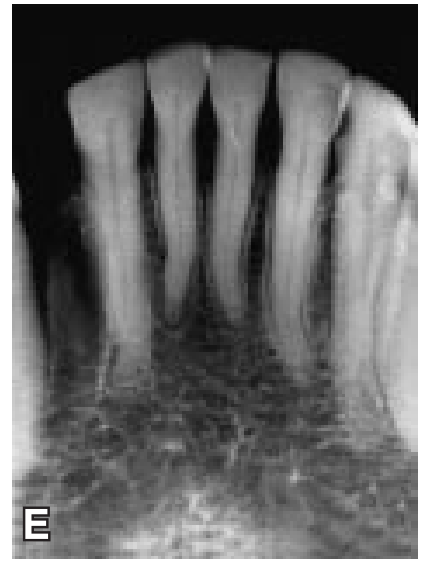

FIGURA 2 - Região de incisivos - seqüência de radiografias digitais: inicial (A); broca $6(\mathrm{~B})$; broca $8(\mathrm{C})$; broca 10 (D) e cortical (E). 
ALMEIDA, S. M. de; BÓSCOLO, F. N.; HAITER NETO, F.; SANTOS, J. C. B. dos. Avaliação de três métodos radiográficos (periapical convencional, periapical digital e panorâmico) no diagnóstico de lesões apicais produzidas artificialmente. Pesqui Odontol Bras, v. 15 , n. 1 , p. 56-63, jan./mar. 2001.
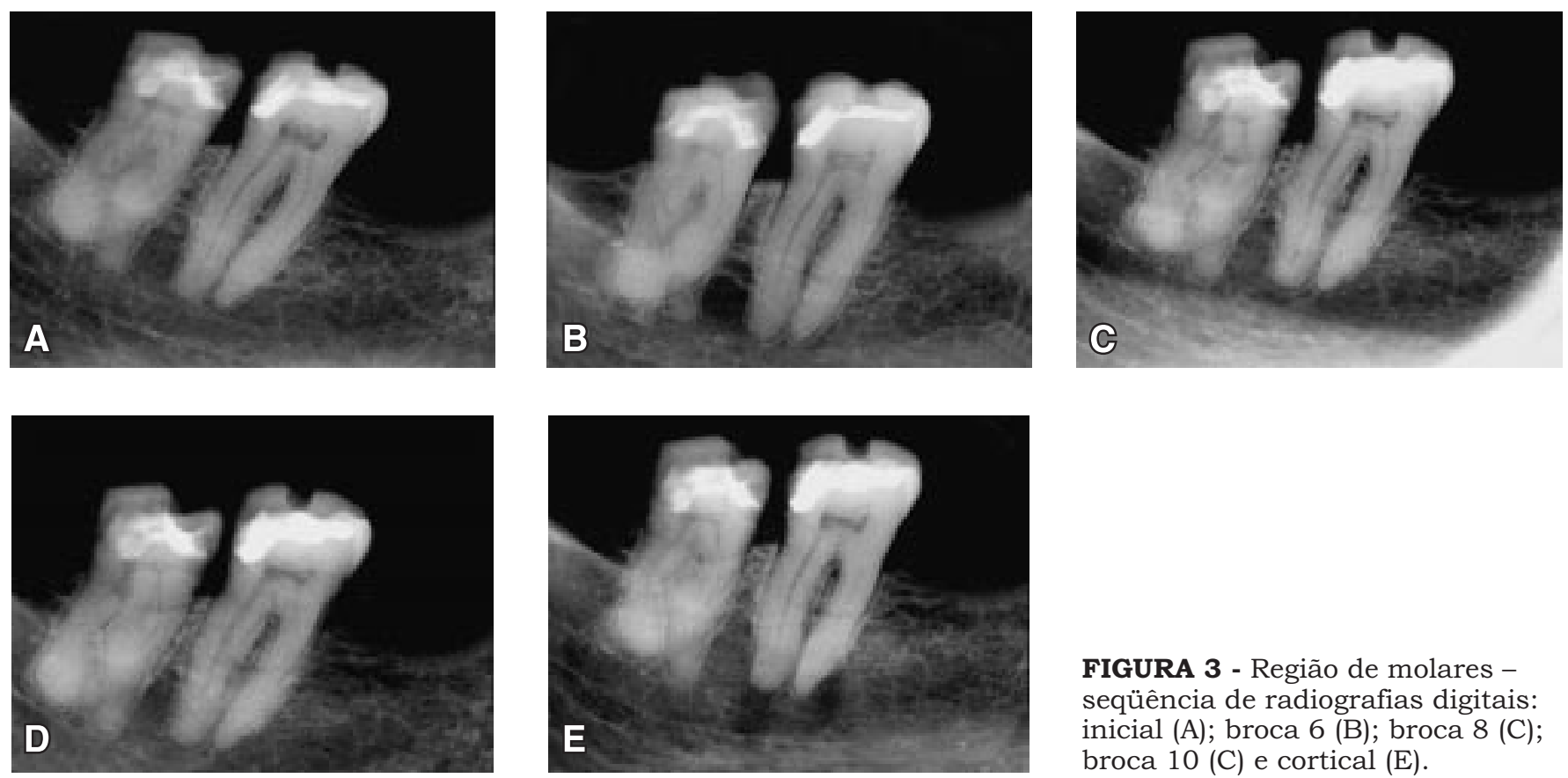

FIGURA 3 - Região de molares seqüência de radiografias digitais: inicial (A); broca $6(\mathrm{~B})$; broca $8(\mathrm{C})$; broca $10(\mathrm{C})$ e cortical $(\mathrm{E})$.

em todas as fases, com exceção da fase inicial em que não havia a presença da lesão, observou-se que a radiografia digital mostrou melhores resultados que a periapical e a panorâmica. Na fase cortical, essa diferença foi estatisticamente significante e, mais uma vez, isso pode ser devido à ampla escala dinâmica desse método e também ao fato de que, uma vez obtida a imagem, essa pode ser manipulada em relação ao contraste ou escala de cinza, o que fornece opções para visualizar nuanças não distinguiveis na radiografia periapical convencional. Resultados semelhantes, quando é realizada a manipulação da imagem, também foi observado no trabalho de KULLENDORFF et al. $^{6}$ (1988) e confirmam a afirmação de WENZEL ${ }^{16}$ (1993) de que a imagem digital é passivel de sofrer alterações, melhorando assim a nitidez dos limites estruturais, diferentemente da radiografia convencional, que apresenta uma imagem estática a partir do momento em que é processada.

Na região dos dentes molares, no que se refere às radiografias periapicais e digitais, pode-se observar uma tendência de aumento na detecção de lesão apical na fase $Z$ (inicial), constituindo um resultado falso-positivo, já que nessa fase ainda não havia lesão apical. Resultados falso-positivos constituem uma probabilidade viável de ocorrência em pesquisas que têm cunho subjetivo de observação, apesar da uniformidade dos avaliadores.
Na fase D (broca 10), houve melhor resultado com as radiografias panorâmicas. Esse mesmo resultado foi observado por PHILLIPS; SHAWKAT ${ }^{10}$ (1973), que o atribuíram ao fato de que os ápices radiculares dos molares estão bem próximos ao plano de corte do aparelho panorâmico utilizado, o que parece bastante lógico, visto que as estruturas radiografadas por essa técnica estarão mais nítidas se estiverem dentro do plano de corte do aparelho. Como a lesão não atingiu a cortical óssea, o ponto médio entre as corticais alveolares da mandíbula correspondente ao osso esponjoso encontrava-se no centro da região de corte do aparelho panorâmico e, portanto, a imagem apresentava-se apenas com a distorção inerente ao método, mostrando limites mais nítidos.

$\mathrm{Na}$ mesma região, quando se analisa a fase $\mathrm{H}$ (cortical óssea), observa-se que a radiografia periapical e a radiografia digital apresentaram melhores resultados no diagnóstico da lesão quando comparadas à radiografia panorâmica. A explicação pode ser a mesma mencionada acima.

Embora os resultados demonstrem serem as três técnicas similares, observa-se que na técnica digital, embora a dose de radiação seja menor em relação à radiografia periapical, não há comprometimento do diagnóstico final. Observa-se também que a radiografia panorâmica, em algumas cir- 
ALMEIDA, S. M. de; BÓSCOLO, F. N.; HAITER NETO, F.; SANTOS, J. C. B. dos. Avaliação de três métodos radiográficos (periapical convencional, periapical digital e panorâmico) no diagnóstico de lesões apicais produzidas artificialmente. Pesqui Odontol Bras, v. 15 , n. 1, p. 56-63, jan./mar. 2001.

cunstâncias, é um método confiável de diagnóstico de lesões apicais.

\section{CONCLUSÕES}

1. Os três métodos radiográficos avaliados demonstraram resultados muito próximos no diagnóstico de lesões apicais; entretanto, a radiografia digital mostrou-se estatisticamente superior aos outros, na região dos dentes incisivos na fase $\mathrm{R}$ e na região de pré-molares na fase $\mathrm{H}$. Na região de molares, o melhor método para o diagnóstico precoce de lesões apicais foi, na fase $\mathrm{H}$, a radiografia panorâmica e, na fase $\mathrm{D}$, a radiografia digital. Na região de caninos, embora não estatisticamente significante, a técnica periapical foi a eleita em todas as fases.

2. O aumento nas dimensões das lesões propicia um melhor diagnóstico radiográfico, mesmo antes de tocar a cortical óssea.

ALMEIDA, S. M. de; BÓSCOLO, F. N.; HAITER NETO, F.; SANTOS, J. C. B. dos. Evaluation of three radiographic methods (conventional periapical, digital periapical and panoramic) in the diagnosis of artificially produced periapical lesions. Pesqui Odontol Bras, v. 15, n. 1, p. 56-63, jan./mar. 2001.

This research was carried out in order to evaluate three radiographic methods - conventional periapical, digital periapical and panoramic - in the diagnosis of artificially produced periapical lesions. For this purpose, 5 mandibles, with lesions produced by means of spherical drills of different sizes, were used. The research was divided into five distinct phases, as follows: phase $Z$ (initial) - characterized by the absence of lesion; phase $\mathrm{R}$ - lesion produced with a number 6 drill; phase $\mathrm{J}$ - lesion produced with a number 8 drill; phase $\mathrm{D}$ - lesion produced with a number 10 drill; and phase $\mathrm{H}$ - lesion reaching the vestibular cortex. The lesions were produced in quadrants. Radiographs were made after each phase and analyzed by 4 experts in radiology. For the digital system there was statistically significant difference in phase $\mathrm{R}$ (in the region of incisors) and in phase $\mathrm{H}$ (in the region of premolars). In the region of molars there was statistically significant difference in phase $\mathrm{D}$ for panoramic radiography. It must be pointed out that panoramic radiography produced the less effective results in phase $\mathrm{H}$.

UNITERMS: Periapical abscess; Radiographic image enhancement; Radiography, panoramic; Radiography, dental.

\section{REFERÊNCIAS BIBLIOGRÁFICAS}

1. BARBAT, J.; MESSER, H. H. Detectability of artificial periapical lesions using direct digital and conventional radiography. J Endod, v. 24, n. 12, p. 837-842, Dec. 1998.

2. DAMANTE, J. H.; CARVAlHO, P. V. Contribuición a la interpretación radiográfica de lesiones oseas producidas experimentalmente en mandíbulas humanas secas. Revi Odontol Dominicana, v. 2, n. 3, p. 49-57, jul./dez. 1993.

3. GRÖNDAHL, H. G.; WENZEL, A.; BORG, E.; TAMMISALO, E. An image plate system for digital intra-oral radiography. Dent Update, v. 23, n. 8, p. 334-337, Oct. 1996.

4. HUDA, W.; RILL, L. N.; BENN, D. K.; PETTIGREW, J. C. Comparison of a photostimulable phosphor system with film for dental radiology. Oral Surg Oral Med Oral Pathol Oral Radiol Endod, v. 83, n. 6, p. 725-731, June 1997.

5. KULLENDORFF, B.; NILSSON, M. Diagnostic accuracy of direct digital dental radiography for the detection of periapical bone lesions. Oral Surg Oral Med Oral Pathol Oral Radiol Endod, v. 82, n. 5, p. 585-589, Nov. 1996.

6. KULLENDORFF, B.; GRÖNDAHL, K.; ROHLIN, M. et al. Subtraction radiography for the diagnosis of periapical bone lesions. Endod Dent Traumatol, v. 4, n. 6, p. 253-259, Dec. 1998.

7. MARMARY, Y.; KOTER, T.; HELING, I. The effect of periapical rarefying osteitis on cortical and cancellous bone. A study comparing conventional radiographs with computed tomography. Dentomaxillofac Radiol, v. 28, n. 5, p. 267-271, Sept. 1999.

8. PAULS, V.; TROTT, J. R. A radiological study of experimentally produced lesions in bone. Dent Practit, v. 16, p. 254-258, 1966.

9. PAURAZAS, S. B.; GEIST, J. R.; PINK, F. E. et al. Comparison of diagnostic accuracy of digital imaging by using CCD and CMOS-APS sensors with E-speed film in the detection of periapical bony lesions. Oral Surg Oral Med Oral Pathol Oral Radiol Endod, v. 89, n. 3, p. 356-362, Mar. 2000.

10. PHILLIPS, J. D.; SHAWKAT, A. H. A study of the radiographic appearance of osseous defects on panoramic and conventional films. Oral Surg, v. 36, n. 5, p. 745-749, Nov. 1973.

11. ROHLIN, M.; KULLENDORFF, B.; AHLQWIST, M. et al. Comparison between panoramic and periapical radiography in the diagnosis of periapical bone lesions. Dent Maxillo Facial Radiol, v. 18, n. 4, p. 151-155, Nov. 1989.

12. SCARFE, W. C.; CZERNIEJEWSKI, V. J.; FARMAN, A. G. et al. In vivo accuracy and reliability of color-coded image enhancements for the assessment of periradicular lesion. Oral Surg Oral Med Oral Pathol Oral Radiol Endod, v. 88, n. 5, p. 603-611, Nov. 1999.

13. SELTZER, S.; BENDER, I. B. Roentgenographic and direct 
ALMEIDA, S. M. de; BÓSCOLO, F. N.; HAITER NETO, F.; SANTOS, J. C. B. dos. Avaliação de três métodos radiográficos (periapical convencional, periapical digital e panorâmico) no diagnóstico de lesões apicais produzidas artificialmente. Pesqui Odontol Bras, v. 15, n. 1, p. 56-63, jan./mar. 2001.

observation of experimental lesions in bone - I. J Am Dent Assoc, v. 62, p. 152-160, Feb. 1961.

14. SELTZER, S.; BENDER, I. B. Roentgenographic and direct observation of experimental lesions in bone - II. J Am Dent Assoc, v. 62, p. 708-716, June 1961.

15. SHROUT, M. K.; HALL, J. M.; HILDEBOLT, C. E. Differentiation of periapical granulomas and radicular cysts by digital radiometric analysis. Oral Surg, v. 76 , n. 3, p. 356-361, Sept. 1993.
16. WENZEL, A. Computer-aided image manipulation of intraoral radiographs to enhance diagnosis in dental practice: a review. Int Dent J, v. 43, n. 2, p. 99-108, Apr. 1993.

17. YOKOTA, E. T.; MILES, D. A.; NEWTON, C. W. et al. Interpretation of periapical lesions using RadioVisioGraphy. J Endod, v. 20, n. 10, p. 490-494, Oct. 1994.

\begin{tabular}{|c|c|c|}
\hline 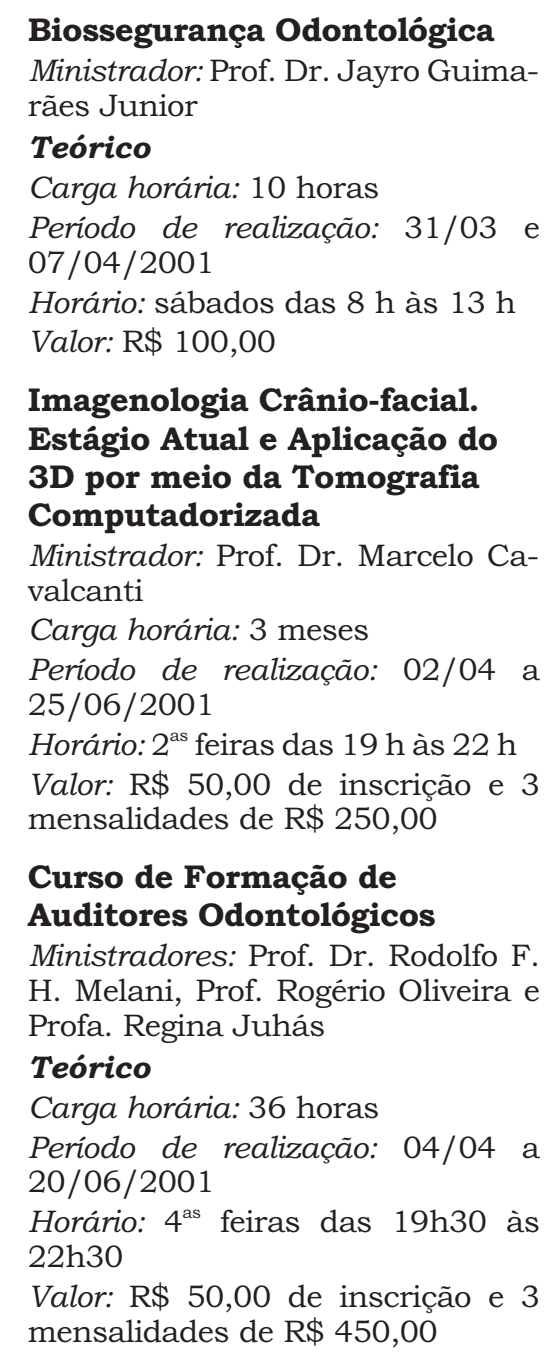 & $\begin{array}{l}\text { Atualização em Dentistica } \\
\text { Ministradores: Profa. Dra. Eliza } \\
\text { Maria Agueda Russo e equipe } \\
\text { Teórico } \\
\text { Duração do curso: } 12 \text { horas } \\
\text { Periodo de realização: 04, } 11 \text { e } \\
18 / 05 / 2001 \\
\text { Horário: } 6^{\text {as }} \text { feiras das } 19 \text { h às } 23 \text { h } \\
\text { Valor: } \$ \$ \text { 100,00 } \\
\text { Diagnóstico e Tratamento do } \\
\text { Ronco e da Apnéia Obstrutiva } \\
\text { do Sono } \\
\text { Coordenadora: Profa. Dra. Beatriz } \\
\text { Silva Câmara Mattos } \\
\text { Ministradores: Dr. Weber P. Cardo- } \\
\text { so, Dr. Ademir Baptista e Silva, Dr. } \\
\text { Domenico Modesto Júnior e Dra. } \\
\text { Dilma Simões } \\
\text { Teórico } \\
\text { Duração do curso: } 6 \text { horas } \\
\text { Periodo de realização: } 05 / 05 / 2001 \\
\text { Horário: sábado das } 9 \text { h às } 17 \text { h } \\
\text { Valor: R\$ } 50,00 \\
\text { Endodontia para Especialistas } \\
\text { Ministradores: Prof. Dr. João Hum- } \\
\text { berto Antoniazzi e Prof. Dr. José } \\
\text { Luiz Lage Marques } \\
\text { Periodo de realização: } 10,11 \text { e } \\
12 / 05 / 2001 \\
\text { Horário: } 5^{a} \text { e } 6^{a} \text { feiras das } 9 \text { h às } \\
18 \text { h e sábado das } 9 \text { h às } 13 \text { h } \\
\text { Duração: } 20 \text { horas } \\
\text { Valor: R } \$ 300,00\end{array}$ & 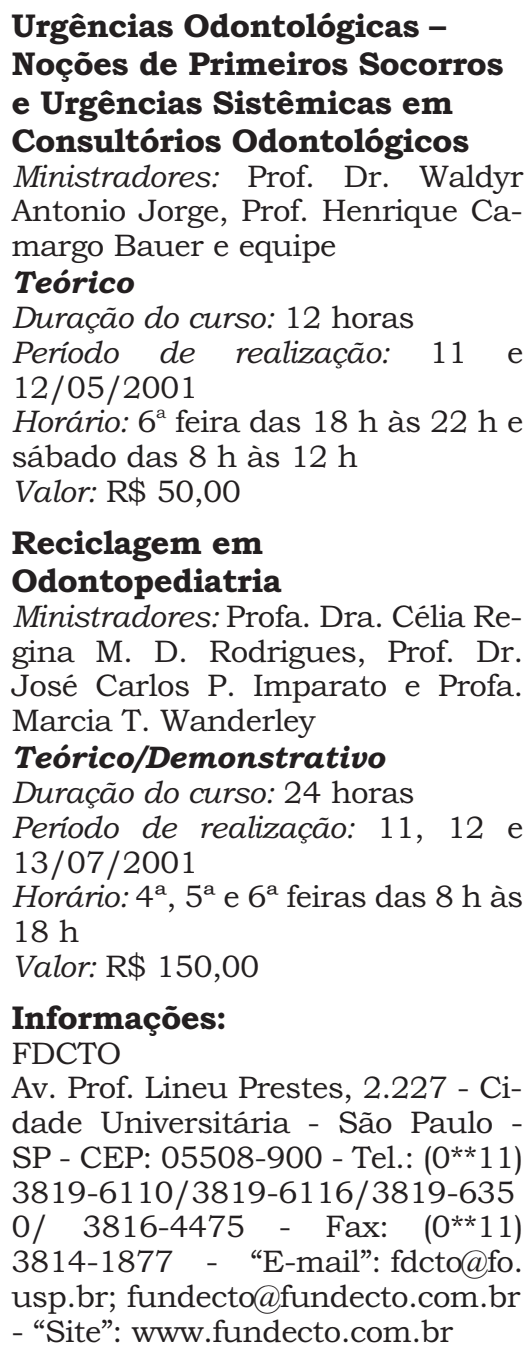 \\
\hline
\end{tabular}

